\title{
Combination of Excimer Laser and Topical Treatment for Psoriasis: A Systematic Review and Meta-analysis
}

\author{
Marie Len A. Camaclang, ${ }^{1}$ Leah Antoinette M. Caro-Chang ${ }^{1}$ and Ma. Lorna F. Frez ${ }^{2}$ \\ ${ }^{1}$ Section of Dermatology, Department of Medicine, Philippine General Hospital, University of the Philippines Manila \\ ${ }^{2}$ Section of Dermatology, Department of Medicine, College of Medicine and Philippine General Hospital, University of the Philippines Manila
}

\begin{abstract}
Objectives. To assess the efficacy and safety of excimer laser in combination with topical standard therapies for treatment of plaque-type psoriasis in comparison to excimer laser alone, standard topical treatment alone, or placebo.
\end{abstract}

Methods. A literature search using Medline, Cochrane and HERDIN was conducted. Data were analyzed using mean difference at $95 \%$ confidence interval, with heterogeneity determined by $\mathrm{I}^{2}$ test.

Results. Three articles with total of 130 patients fulfilled the inclusion criteria. Topical treatments studied were vitamin analog (calcipotriol), anthralin (dithranol), and steroid (flumethasone pivalate). A subgroup analysis comparing combination therapy and excimer laser alone showed a greater reduction in pooled PASI score reduction (-2.52; $95 \% \mathrm{Cl}:-4.28,-0.77)$ in the combination group after five to six weeks. There was also a significantly greater reduction in cumulative UVB dose $(-3.29 ; 95 \% \mathrm{Cl}$ : $-4.29,-2.30)$ needed for clearing in the combination group. Pigmentation was the commonly observed adverse event in both groups.

Conclusions. Excimer laser, in combination with topical treatment, is more effective than excimer laser alone, with significantly lower cumulative UVB dose, but the quality of current evidence is low. Long-term controlled trials are warranted to increase our confidence in the estimates of these outcomes.

Key Words: excimer, psoriasis, combination therapy, calcipotriol, dithranol, flumethasone, meta-analysis, systematic review

\section{INTRODUCTION}

Poster presented at the $21^{\text {st }}$ Philippine General Hospital (PGH) Annual Research Week, November 7-11, 2016, PGH, Manila, Philippines.

E-poster presented at the $39^{\text {th }}$ Annual Convention of the Philippine Dermatological Society, November 3-5, 2016, EDSA Shangri-La Hotel, Mandaluyong City, Philippines.

Corresponding author: Marie Len A. Camaclang, MD

Section of Dermatology

Department of Medicine

Philippine General Hospital

University of the Philippines Manila

Taft Avenue, Manila 1000, Philippines

Telephone: +632 5548400 local 5105/5106

Email: len.camaclang@gmail.com
Psoriasis is a chronic and relapsing condition that affects approximately $2-3 \%$ of the world's population. ${ }^{1}$ The most common type is plaque psoriasis, and is primarily diagnosed clinically by the presence of erythematous plaques with silvery scales, with a predilection for extensor surfaces such as knees and elbows. ${ }^{2}$ Management is geared towards controlling the disease with different treatment modalities which include topical and systemic medications. Despite having immunosuppressive properties, these interventions are not without adverse effects, especially on extended duration of treatment. Hence, there is a continuing challenge to search for effective and safe treatment options, possibly long-term, that target the underlying immunologic mechanisms of the disease. ${ }^{3}$

One viable long-term intervention with attributed immunosuppressive properties is phototherapy. Treatment with UV light is commonly employed in various skin disorders and it remains to be one of the safest and one of the least costly interventions for psoriasis. ${ }^{4}$ However, standard units using cabinets or panels do not spare normal, uninvolved skin from UV radiation which can theoretically lead to photoaging and higher incidence of skin cancer. 
Considering the side effect of whole-body phototherapy, the role of targeted phototherapy, where only psoriatic lesions are irradiated, has been increasingly explored. In a study by Parrish and Jaenicke, ${ }^{5}$ a 300-313 nm spectrum of wavelength was shown to be most significant in the treatment of psoriasis. In a recent systematic review, ${ }^{6}$ among the targeted UVB phototherapy, the efficacy of excimer laser is $70 \%$ compared to $59 \%$ for excimer light and $49 \%$ for localized NB-UVB. Excimer laser is a type of UVB phototherapy that transmits a beam of light through xenon-chloride gas, with a $308 \mathrm{~nm}$ wavelength, using a fiberoptic arm that allows selective skin irradiation of psoriatic plaques. ${ }^{7}$ The device uses a limited spot diameter of 14-30 mm. ${ }^{3}$ Excimer lasers act by inhibiting the generation of inflammatory cytokines and by inducing apoptosis of $\mathrm{T}$ cells. ${ }^{8}$ The latter mechanism is attributed to the upregulation or stabilization of $\mathrm{p} 53$, an anti-oncogenic molecule. ${ }^{9}$ Traditional phototherapy requires approximately two to three weekly sessions for three months or a total of 24-36 treatments. ${ }^{10}$ Excimer laser as monotherapy, on the other hand, was shown to reduce the range of sessions to approximately 8-12 treatments. ${ }^{11}$

The therapeutic use of excimer laser in psoriasis was first demonstrated in the 1997 study by Bonis et al. ${ }^{12}$ Excimer laser is one of the localized phototherapies recommended by the American Academy of Dermatology for patients with less than $10 \%$ affected body surface area. ${ }^{13}$ It has, however, in recent studies, shown to be efficacious even in generalized psoriasis. ${ }^{10,14}$ However, more extensive body involvement entails greater UV exposure. Accumulated cumulative dose plays a crucial role in increasing adverse effects from UV exposure. ${ }^{15}$ Studies suggest that combination therapies may provide greater therapeutic benefit by enhancing efficacy and decreasing cumulative dose needed for treatment. ${ }^{14}$ A plausible mechanism is the greater laser penetration provided by topical therapies by decreasing the high airtissue interface caused by a thickened stratum corneum. ${ }^{16}$ Thus, combination therapies further expand the potential of excimer laser for long-term treatment by optimizing efficacy while minimizing side effects. To date, there is no existing published systematic review or meta-analysis exploring combination therapies with excimer laser, despite the growing body of research on their potential therapeutic benefits.

This study assessed the efficacy and safety of excimer laser in combination with topical standard therapies for the treatment of psoriasis in comparison to excimer laser alone, standard topical treatment, or placebo.

\section{METHODS}

\section{Selection criteria}

\section{Type of studies}

Randomized controlled trials (RCTs) comparing combination therapy of excimer laser and topical treatment (corticosteroids, anthralin, vitamin $\mathrm{D}$ analogs, tar), with excimer laser as monotherapy, placebo, or standard topical treatment for plaque-type psoriasis were included. No exclusions were made with regard to publication status, sample size, or language. When original articles were written in non-English language, we included them if English-translated articles were available. Duplicates of already-included studies were excluded. Included studies must have outcome measures for clinical efficacy and safety as primary or secondary outcomes.

\section{Types of participants}

We included all adult patients who were clinically diagnosed with plaque-type psoriasis. No exclusions were made on duration and severity of condition. Other types of psoriasis (e.g. nail, palmoplantar, pustular, and guttate) were excluded.

\section{Types of interventions}

Studies included were trials that compared excimer laser combined with any standard topical treatment for psoriasis (e.g. corticosteroids, anthralin, vitamin D analog, or tar) versus excimer laser alone, standard topical treatment, or placebo. No exclusions were made on the basis of duration of treatment. Trials which used excimer laser combined with more than one standard topical therapy for psoriasis were permitted.

\section{Types of outcome measures}

Outcome measures for efficacy included clinical improvement and/or clinical cure at the end of intervention. Clinical improvement refers to reduction in signs namely, erythema, scaling, and/or infiltration of lesions (e.g. mean reduction in PASI). Clinical cure refers to complete clearance of lesions by using objective standard scoring (e.g. PASI).

Outcome measures for safety included adverse events which are any new symptoms during the period of intervention, as reported by the participants or as inquired by the researchers. Safety outcome also included the mean cumulative dose of MED used at the end of study period.

\section{Search strategy}

Electronic databases namely Medline, The Cochrane library and HERDIN using relevant terms namely, "psoriasis", "excimer laser," "excimer," "targeted UVB," and "targeted phototherapy," were searched up to May 2016 for reviews and clinical trials. The PROSPERO (International Prospective Register of Systematic Reviews) was searched to identify existing reviews. Reference lists of identified studies were also perused for clinical trials and relevant reviews.

\section{Data collection and analysis}

\section{Data extraction and management}

Studies were selected by consensus of three independent review authors. The full-text articles were 
reviewed using a standard eligibility form patterned after the data extraction table from Review Manager (v. 5.3). Data regarding dropouts and funding sources were also recorded. Appraisal of each journal was independently done by two review authors. A third review author was consulted to resolve any discrepancies or disagreements between the two independent authors.

\section{Data synthesis and analysis}

Statistical analysis was done using the Review Manager software (version 5.3) provided by The Cochrane Collaboration. Data were pooled using random-effects models and to obtain weighted effect across trials. Metaanalyses were done using the software package Review Manager 5.3 (RevMan 2014). Heterogeneity was visually assessed using the forest plot and objectively determined using the $\mathrm{I}^{2}$ statistic.

\section{Quality assessment}

Internal validity of included trials was independently assessed by two review authors using the Cochrane risk of bias tool. The tool employed criteria outlined in the Cochrane Handbook for Systematic Reviews of Interventions. Any discrepancies were settled by discussion between the authors, and resolved by a third party. The GRADE approach to assessing quality of evidence was used. ${ }^{17}$

\section{RESULTS}

A total of 183 records through the electronic searches from The Cochrane Library $(\mathrm{n}=25), \operatorname{MEDLINE}(\mathrm{n}=158)$ was obtained. No local trials were identified from HERDIN. The study flow diagram is shown in Figure 1.

\section{Study characteristics}

Three randomized controlled trials involving 130 patients clinically diagnosed with stable plaque-type psoriasis were included in this review. The topical treatments used in the studies were vitamin analog (calcipotriol), anthralin (dithranol), and steroid (flumethasone pivalate). The three studies which fulfilled the inclusion criteria are summarized in Table 1. Three of the full-text articles screened were excluded (Table 2). Although all the studies used a combination therapy of excimer laser and topical treatment for psoriasis, they did not qualify mainly because of their study design-one was a case report (Kardorff 2013), while the other was an open, self-control trial (Fritz 2007). One randomized controlled trial was excluded because it used three different treatment protocols in a total of 12 weeks (Levin 2015).

In all studies, the primary outcome was primarily measured by adopting the Psoriasis Area Severity Index (PASI) measured at baseline and at the end of the study period. One study used a modified PASI or the Psoriasis Severity Index (PSI) and excluded Area (A) as part of the scoring because the unit of analysis for this study was each

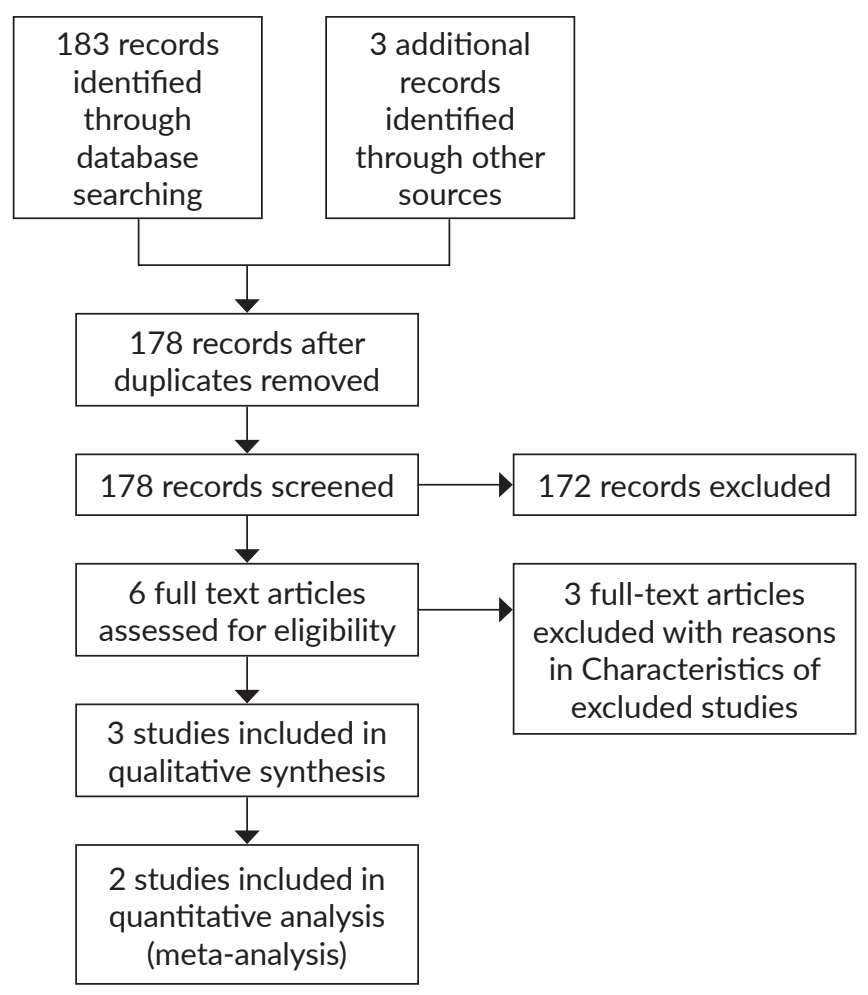

Figure 1. Study flow diagram of literature selection according to PRISMA.

individual psoriatic lesion (Rogalski 2012). Hence, only the Dong 2012 study and Tang 2014 study had comparable data for the primary outcome available for meta-analysis.

\section{Outcomes reported}

\section{Primary outcomes}

Table 3 shows summary of primary outcomes for the three included studies. The Rogalski 2012 study used dichotomous outcomes (clinical response divided into partial clearance and total clearance), while the Dong 2012 study used continuous outcome (mean PASI). The Tang 2014 study used both continuous (mean PASI) and dichotomous (clinical efficacy) outcomes in presenting the results.

Clinical improvement: The primary outcome measured in all studies was clinical improvement by documenting reduction in mean PASI scores at the end of the study period from baseline measurement. The Tang 2014 study showed significant difference in PASI scores at the end of 6 weeks between the combination therapy and excimer laser alone. In addition, clinical efficacy of $>60 \%$ to $>95 \%$ was considered effective. For the Rogalski 2012 study which used modified PASI scoring (PSI), combination therapy showed significant difference compared to topical treatment alone. In both studies, combination therapy showed significant response at the end of the study duration, with combination of dithranol and excimer laser having slightly better response in one trial (Rogalski 2012). 
Table 1. Characteristics of included studies using the PICOM format

\begin{tabular}{|c|c|c|c|}
\hline & Tang 2014 & Dong 2012 & Rogalski 2012 \\
\hline \multicolumn{4}{|l|}{ Participants } \\
\hline Total & 36 & 40 & 54 \\
\hline Age & $20-60$ yrs (32.56 / 10.48) & $22-60$ years & $23-77(45.6 / 10.7)$ \\
\hline Gender & $\begin{array}{c}21 \text { males } \\
15 \text { females }\end{array}$ & $\begin{array}{l}21 \text { males } \\
19 \text { females }\end{array}$ & $\begin{array}{l}38 \text { males } \\
16 \text { females }\end{array}$ \\
\hline Diagnosis & $\begin{array}{c}\text { Plaque psoriasis } \\
<30 \% \text { BSA }\end{array}$ & $\begin{array}{l}\text { Plaque psoriasis } \\
\text { Mild-mod } \\
<50 \% \text { BSA }\end{array}$ & $\begin{array}{c}\text { Plaque psoriasis } \\
\text { PASI 1.2 or higher in all plaques } \\
\text { (total of }>=6 \text { ) }\end{array}$ \\
\hline Country & China & China & Germany \\
\hline Intervention & $\begin{array}{c}\text { excimer laser } 2 x / \text { week + } \\
\text { calcipotriene ointment (0.005\%) BID }\end{array}$ & $\begin{array}{c}\text { excimer laser } 2 x / \text { week }+ \\
\text { flumethasone (flumethasone } \\
\text { pivalate } 0.2 \mathrm{mg}+\text { SA } 30 \mathrm{mg} \text { ) BID }\end{array}$ & $\begin{array}{c}\text { 1st: excimer laser 2x/week } \\
\text { cacipotriol 3\% ointment BID } \\
\text { 2nd: + excimer laser 2x/week + } \\
\text { dithranol ointment 3\% OD + SA 5\% }\end{array}$ \\
\hline Comparator & excimer laser $2 x /$ week & excimer laser $2 x /$ week & $\begin{array}{c}3^{\text {rd }}: 3 \% \text { calcipotriol BID } \\
4^{\text {th }} \text { dithranol OD }\end{array}$ \\
\hline Outcome/s & $\begin{array}{l}\text { PASI score difference } \\
\text { \# of irradiation } \\
\text { Cumulative dose } \\
\text { Side effects }\end{array}$ & $\begin{array}{c}\text { PASI score difference } \\
\text { Cumulative dose } \\
\text { Side effects } \\
\text { recurrence rate } \\
\text { Patient-reported outcome on } \\
\text { treatment efficacy and degree } \\
\text { of satisfaction (excellent, good, } \\
\text { moderate, poor) - no data }\end{array}$ & Modified PASI score \\
\hline Duration & 6 weeks & 5 weeks & 8 weeks \\
\hline Assessment timing & After 2,4 and 6 weeks & After 10 treatments or 5 weeks & After 8 weeks \\
\hline Follow up & & at 3 and 6 months after completion & At 4 and 6 months after completion \\
\hline Methods/Design & RCT, open and parallel self-control & RCT & RCT, self-control \\
\hline Remarks & Supported by Pharmaceutical company & Supported by Pharmaceutical company & With research grant \\
\hline
\end{tabular}

a Population, Intervention, Comparison, Outcome, Methods

Table 2. Characteristics of excluded studies

\begin{tabular}{|c|c|c|c|}
\hline & Kardoff 2003 & Fritz 2007 & Levin 2015 \\
\hline Design & RCT, self-control & Open trial, self-control & \\
\hline Participants & $\begin{array}{l}8 \\
\text { Plaque-type }\end{array}$ & $\begin{array}{l}36 \\
\text { Mod-severe } \\
\text { Plaque-type }\end{array}$ & $\begin{array}{l}30 \\
10-20 \% \text { BSA } \\
\text { Plaque-type }\end{array}$ \\
\hline Intervention & $\begin{array}{l}\text { 1. Calcipotriol BID + Excimer } \\
\text { 2. Excimer }\end{array}$ & $\begin{array}{l}\text { 1. Calcipotriol ointment BID } \\
\text { 2. Excimer + Vaseline }\end{array}$ & $\begin{array}{l}\text { Duration: } 12 \text { weeks } \\
\text { 1. Clob spray on } 1^{\text {st }} 4 \text { weeks } \\
\text { 2. Calcipotriol ointment } \\
\text { 3. Calcipotriol + Clob +excimer wk 9-12 }\end{array}$ \\
\hline Outcome & $\begin{array}{l}\text { Modified PASI (PSI) } \\
\text { \# of sessions }\end{array}$ & PASI & $\begin{array}{l}\text { PASI } \\
\text { PGA } \\
\text { DLQI/Koo-Menter Psoriasis Assessment }\end{array}$ \\
\hline Reason for exclusion & Only presented a case report & Not an RCT & $\begin{array}{l}\text { Study used two treatment protocols with } \\
\text { combination therapy consecutively }\end{array}$ \\
\hline
\end{tabular}

Table 3. Primary outcomes of included studies with combination therapy of excimer laser and standard topical treatment as intervention

\begin{tabular}{llll}
\hline & Tang 2014 & Dong 2012 & Rogalski 2012 $^{\text {a }}$ \\
\hline Study duration & 6 weeks & 5 weeks & 8 weeks \\
\hline Clinical Improvement & Mean PASI reduction & Mean PASI reduction ${ }^{\mathrm{c}}$ & 'Partial clearance' (PSI of > 2) \\
& 'Clinical efficacy' & & Reduction of PSI \\
\hline Clinical Cure & Not stated & Not stated & 'Total clearance' (PSI of 0) \\
\hline
\end{tabular}

a Used dichotomous outcomes (frequency and percentage distribution);

${ }^{b}$ Clinical efficacy measured as total number of 'effective treatment' (>60\%-95\% decrease in PASI) and 'recovery' (>95\% decrease in PASI)

c Used direct and polarized light photographs

Clinical cure: In the Rogalski 2012 study, clinical cure was defined as a total clearance or modified PASI score of 0 at the end of 8 weeks. The groups with combination of topical treatment and excimer laser had total clearance
(6.8\% with calcipotriol and $10.2 \%$ with dithranol) while the groups without laser treatment failed to achieve total clearance at the end of 8 weeks. In another study (Tang 2014 study), PASI reduction of $>95 \%$ from baseline was 
regarded as recovery. The combination laser and topical treatment group showed significantly greater efficacy (84.37\%) compared to control group (56.25\%). No data was given on the number and proportion who achieved PASI of 0 at the end of the study.

\section{Secondary outcomes}

Cumulative dose: Data for cumulative UVB dose were present in two studies (Tang 2014, Dong 2012) which showed significantly lower mean for the treatment group compared to laser group. Only the total average cumulative UVB dose was presented in the study by Rogalski (2012), and no categorization was done for each group receiving excimer laser.

Adverse events: Two of the included studies (Tang 2014, Dong 2012) showed data on adverse events. However, only the latter study compared adverse events in the treatment and control group whereas the former narrated the total side effects, wherein 6/32 (18.8\%) showed adverse events during the study. Pigmentation was commonly observed in both studies. In the Dong 2012 study, pigmentation was observed after five sessions in most areas, occurring earlier in combination treatment, which diminished with follow up at 6 months after treatment. Burning sensation. erythema, pruritus, and blister formation were the other reported side effects in decreasing order. Blister formation was noted to be temporary and in the trial by Dong 2012 study, disappeared after 3-5 days, and only occurred in the group receiving excimer laser alone.

\section{Pooled analyses}

Two studies were included in the meta-analysis (Dong 2012, Tang 2014). These studies compared combination therapy and excimer laser alone. There was a greater reduction in pooled PASI score -2.52 (95\% CI: -4.28 , -0.77) with large effect size in the combination therapy group compared to excimer laser alone at the end of the study period (Figure 2). Due to high heterogeneity, the random-effect model was the chosen effect measure used and illustrated. The high heterogeneity $\left(\mathrm{I}^{2}=74 \%\right)$ suggests that the intervention may not consistently have a large effect.

Safety of the intervention was primarily measured by cumulative UVB dose. There was also a significantly greater reduction in cumulative UVB dose score -3.29 (95\% CI: $-4.29,-2.30)$ with large effect size in the combination therapy group compared to excimer laser alone at the end of the study period. In contrast to the first outcome, there was low heterogeneity $\left(\mathrm{I}^{2}=24 \%\right)$ for the pooled mean of cumulative UVB dose (Figure 3 ).

\section{Risk of Bias and GRADE}

None of the included trials were of low risk of bias. Based on risk of bias assessment following the criteria by the Cochrane Collaboration, the trials were of moderatehigh risk of bias (Figures 4 and 5). In terms of reported outcomes, the quality of evidence was further assessed by exploring inconsistency, indirectness, and imprecision as outlined in the GRADEpro software ${ }^{17}$ (Tables 4 and 5).

\section{DISCUSSION}

The topical treatments used in the studies were vitamin analog (calcipotriol), anthralin (dithranol), and steroid (flumethasone pivalate). Calcipotriol is a synthetic vitamin D3 analog, anthralin is an anthracene derivative while flumethasone is a moderately potent difluorinated diglucocorticoid. The immunosuppressive and anti-proliferative properties of these topical treatments

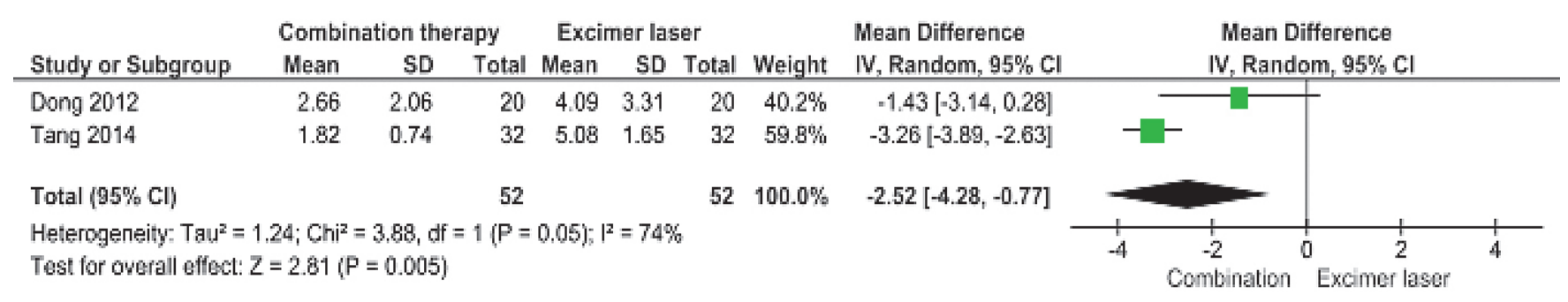

Figure 2. Forest plot of comparison of combination therapy versus excimer laser alone for outcome: PASI score at end of intervention.

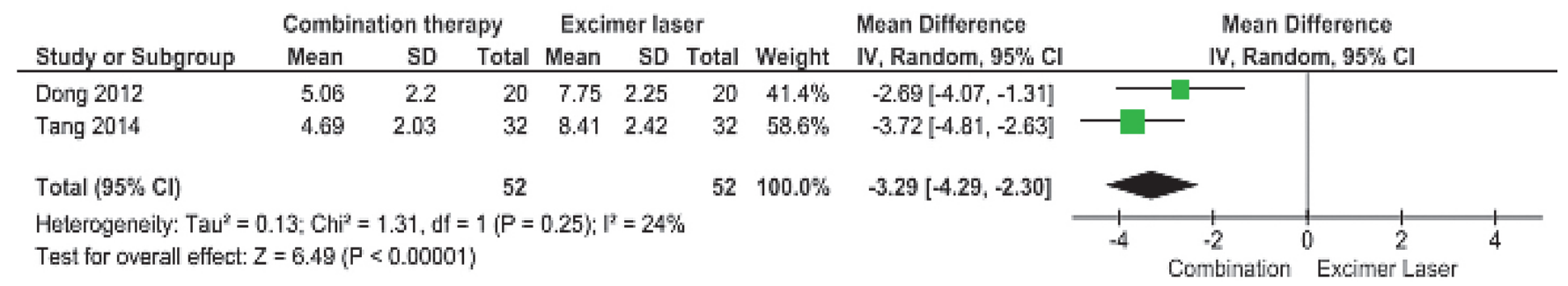

Figure 3. Forest plot of comparison of combination therapy versus excimer laser alone for the outcome: Cumulative UVB dose. 


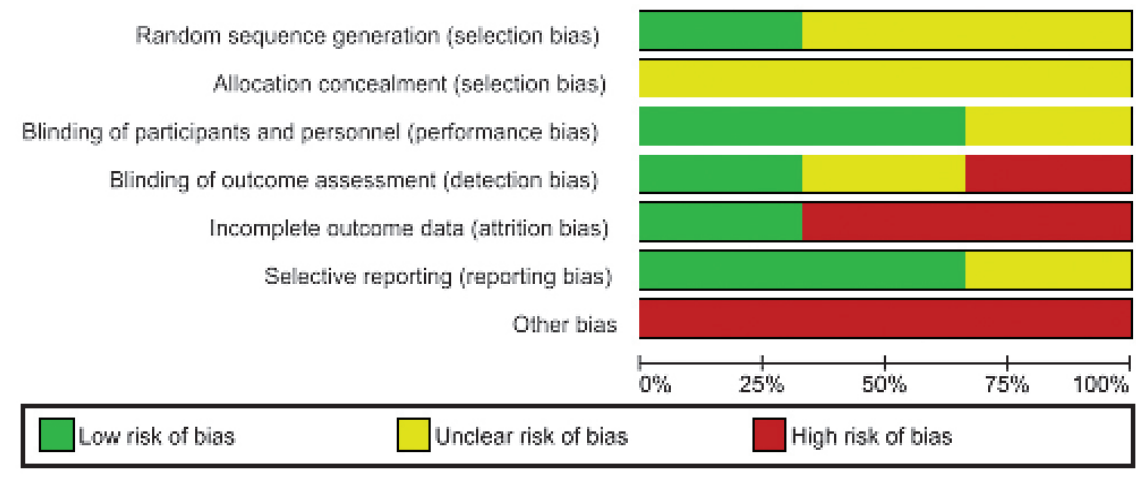

Figure 4. Risk of bias graph: review authors' judgments about each risk of bias item for each included study.

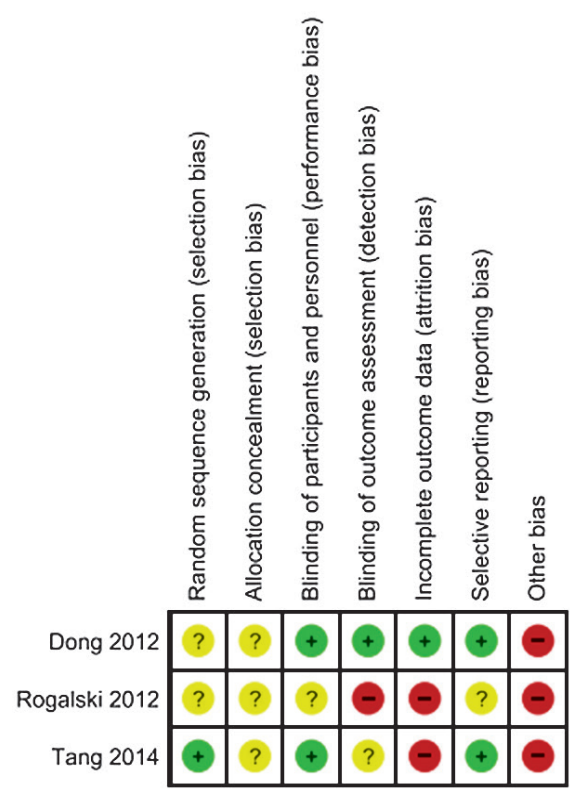

Figure 5. Risk of bias summary: review authors' judgments about each risk of bias item for each included study.

Table 4. Summary of findings

Combination therapy (excimer laser and topical standard treatment) compared with excimer laser therapy alone for psoriasis Patient or population: Adult patients with plaque psoriasis

Intervention excimer laser in combination with topical standard therapies for the treatment of psoriasis

Comparison: excimer laser alone, standard topical treatment for psoriasis or placebo.

\begin{tabular}{|c|c|c|c|c|c|c|}
\hline \multirow[b]{2}{*}{ Outcomes } & \multicolumn{2}{|c|}{ Illustrative comparative risks* $(95 \% \mathrm{Cl})$} & \multirow[b]{2}{*}{$\begin{array}{l}\text { Relative effect } \\
\qquad(95 \% \mathrm{Cl})\end{array}$} & \multirow[b]{2}{*}{$\begin{array}{l}\text { No of participants } \\
\text { (studies) }\end{array}$} & \multirow[b]{2}{*}{$\begin{array}{l}\text { Quality of the } \\
\text { evidence (GRADE) }\end{array}$} & \multirow[b]{2}{*}{ Comments } \\
\hline & $\begin{array}{l}\text { Assumed risk } \\
\text { [excimer laser] }\end{array}$ & $\begin{array}{c}\text { Corresponding risk } \\
\text { [combination] }\end{array}$ & & & & \\
\hline $\begin{array}{l}\text { Cumulative } \\
\text { UVB Dose }\end{array}$ & $\begin{array}{l}\text { The mean cumulative } \\
\text { UVB dose ranged } \\
\text { across control groups } \\
\text { from } 8.08 \mathrm{~J} / \mathrm{cm}^{2}\end{array}$ & $\begin{array}{l}\text { The mean cumulative UVB } \\
\text { dose in the intervention } \\
\text { groups was } 3.29 \text { lower } \\
\text { (2.30-4.29 lower) }\end{array}$ & & [52] (2 studies) & $\bigoplus \oplus \ominus \ominus$ low & \\
\hline Post-PASI & $\begin{array}{l}\text { The mean post- } \\
\text { PASI score ranged } \\
\text { across control groups } \\
\text { from } 4.59 \text {. }\end{array}$ & $\begin{array}{l}\text { The mean post-PASI score } \\
\text { in the intervention groups } \\
\text { was } 2.52 \text { lower } \\
\text { (0.77 to } 4.28 \text { lower] }\end{array}$ & & [52] (2 studies) & $\bigoplus \oplus \oplus \ominus$ moderate & \\
\hline
\end{tabular}

${ }^{*} \mathrm{Cl}$ : Confidence interval

GRADE Working Group grades of evidence: High quality: Further research is very unlikely to change our confidence in the estimate of effect. Moderate quality: Further research is likely to have an important impact on our confidence in the estimate of effect and may change the estimate. Low quality: Further research is very likely to have an important impact on our confidence in the estimate of effect and is likely to change the estimate. Very low quality: We are very uncertain about the estimate.

contribute to their long-term, well-established efficacy and hence, they are regarded as standard topical therapies for psoriasis. They however, possess significant varying side effects that limit extended use such as skin atrophy (steroid), staining (dithranol), and irritant dermatitis (dithranol and calcipotriol).

When compared to excimer laser alone, this review found greater efficacy in the combination group in terms of PASI reduction in 5-6 weeks of treatment. However, the pooled analysis showed insignificant difference with high degree of heterogeneity. Possible causes of heterogeneity may be attributed to the differences in outcome detection. In the Tang 2014 study, which showed significant difference between the two groups at the end of the treatment, a selfcontrol design was employed which would imply lesser variations. On the contrary, the Dong 2012 study had different sets of participants for each group.

In addition, the large effect of combination therapy compared to excimer laser alone cannot be overviewed. The topical treatments enhance the efficacy of excimer laser by potentiating greater penetration of UV light, thereby resulting to a more effective immune system suppression by inducing $\mathrm{T}$ cell apoptosis. ${ }^{16}$ However, considering the low number of total participants and the limited number of available randomized controlled trials, this systematic review may not have the statistical power to detect 


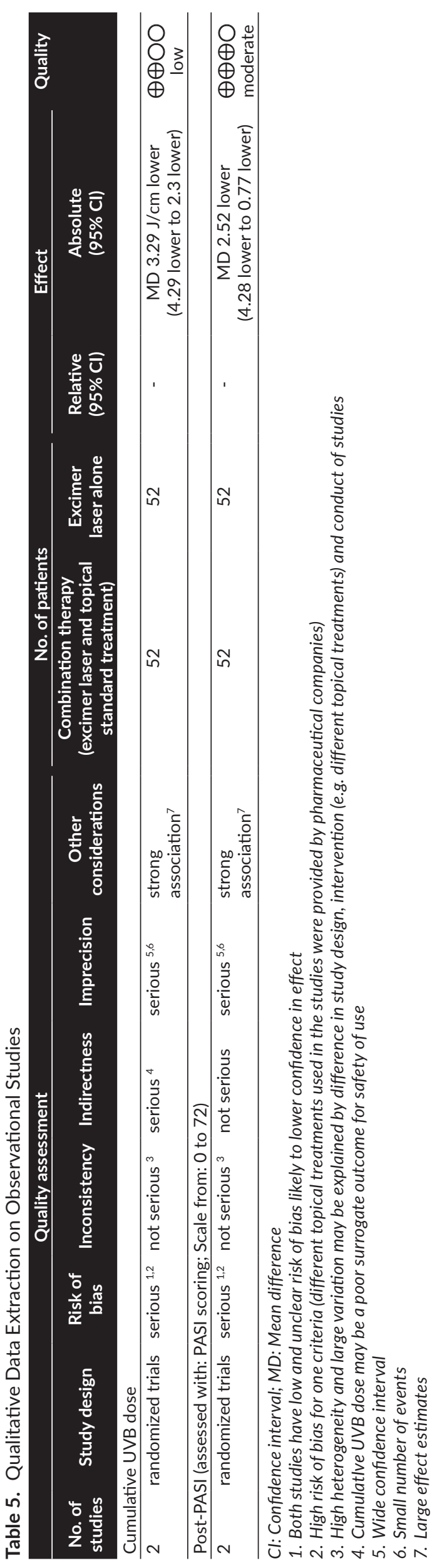


significant difference. Moreover, despite these studies being randomized controlled trials, the high risk of bias is likely to further lower our confidence in its effect.

Safety of the intervention was primarily measured by cumulative UVB dose. There was statistically significant difference between the combination group and excimer laser alone group in the mean cumulative UVB dose at the end of treatment (3.29 fewer (95\% CI 4.29 to 2.30). Both groups received excimer laser twice a week for a total of five to six weeks. The synergistic effect of the topical therapies in terms of anti-proliferation and anti-inflammation reduce the needed intensity of UVB to induce effect, resulting to fewer side effects. ${ }^{18}$ Moreover, the shortened treatment duration seen in the combination group (5 to 6 weeks) compared to traditional phototherapy, which usually takes 24 to 36 treatments (12 weeks), implies a lesser long-term cumulative UVB dose. In a 2014 cross-sectional study among psoriasis patients who underwent UVB therapy of at least 100 times in the last five years, the risk of skin cancer correlated with the number of treatments. ${ }^{19}$ However, despite this finding, cumulative UVB dose may remain a poor surrogate outcome of safety as the over-all risk of skin cancer among the treated patients was not greater than the general population. In terms of adverse events, erythema and blisters were common adverse events seen in localized phototherapy, ${ }^{6}$ but pigmentation was more commonly observed in this study.

Patient-reported outcomes such as cost and quality of life are important outcomes not reported in these trials. Symptoms (e.g. pain, pruritus) were reported under adverse events. However, adverse events as an outcome was only described in two studies (Tang 2014, Dong 2012) and the reporting varied across trials. Thus, subgroup analysis was not done. The number of skin irradiation could be a surrogate outcome for health cost. However, we did not draw any conclusion on the number of skin irradiation because only one study reported this as an outcome.

All of the trials included patients with psoriasis involving a maximum of 50\% BSA. Results, therefore, cannot be generalized to patients suffering from more severe and widespread forms of psoriasis, which would require longer duration of treatment. Despite the growing evidence of excimer laser application in generalized psoriasis, randomized controlled trials on these patients are lacking. In terms of population, majority of the participants in this review were Chinese. Although belonging to Asian descent, the general skin type may have a confounding effect if applied to local setting where a general proportion have skin types III-IV.

The objectives of this systematic review have been addressed primarily by using tools from a clinician's standpoint. Efficacy may also be addressed by using patientreported outcomes, which in this case, was lacking. In terms of safety, cumulative UVB dose may be an unreliable surrogate outcome provided that the studies had limited duration. Patient-reported outcomes of symptoms and of adverse events may be of more benefit for optimal decision-making and future research.

\section{CONCLUSION}

\section{Implication for practice}

The efficacy of excimer laser in combination with topical treatment significantly reduces the PASI score compared to excimer laser alone in 5 to 6 weeks, but the quality of current evidence is low. Combination therapy may be associated with lowered cumulative UVB dose; that is, there is significantly lower cumulative UVB dose in the combination therapy compared to excimer laser alone, but its correlation with safety cannot be deduced. Further research using long-term randomized controlled trials is necessary to increase our confidence in the estimate of effect for these outcomes.

\section{Implication for research}

Future studies should be controlled trials with adequate methods of randomization and allocation concealment. Because severity scoring such as PASI mainly depends on the clinician's standpoint, these trials must employ blinding of outcome assessors. Within-patient analyses may be considered to allow lesser variation and greater statistical power if a large sample size cannot be obtained. A long duration of study which include follow-up and looking at recurrence rates are of benefit in assessing efficacy and safety of the intervention. A standardized protocol for using excimer laser (e.g. initial starting dose, site of initial test site, adjustment principle) to reach endpoints must be clearly described and utilized to limit confounding factors that may affect measurements of cumulative UVB dose. PASI presented as a dichotomous outcome (e.g. $>75 \%$ reduction from baseline) may be more informative and of greater clinical use instead of continuous outcome (mean PASI); in addition, patient-reported outcomes including quality of life and symptoms (e.g. pain) must be emphasized and reported in future trials.

\section{Statement of Authorship}

All authors approved the final version submitted.

\section{Author Disclosure}

All authors declared no conflict of interest.

\section{Funding Source}

This paper was funded by the authors. No external funding agency.

\section{REFERENCES}

1. Kurd SK, Gelfand JM. The prevalence of previously diagnosed and undiagnosed psoriasis in US adults: results from NHANES 2003-2004. J Am Acad Dermatol. 2009;60(2):218-24. doi: 10.1016/j.jaad.2008.09.022.

2. Rogalski C, Grunewald S, Schetschorke M, Bodendorf MO, Kauer F, Simon JC, Paasch U. Treatment of plaque-type psoriasis 
with the $308 \mathrm{~nm}$ excimer laser in combination with dithranol or calcipotriol. Int J Hyperthermia. 2012;28(2):184-90. doi: 10.3109/02656736.2011.647942.

3. Bhutani T, Koo J. The excimer laser for psoriasis: A review of safety and efficacy. Practical Dermatology, 2010;31-6.

4. Hamzavi I, Kerr H. Phototherapy and Laser for the Treatment of Mild-to-Moderate Psoriasis. Mild-to-Moderate Psoriasis, Second Edition. 2008;103-28.

5. Parrish JA, Jaenicke KF. Action Spectrum for Phototherapy of Psoriasis. J Invest Dermatol. 1981;76(5):359-62 .

6. Almutawa F, Thalib L, Hekman D, Sun Q, Hamzavi I, Lim HW. Efficacy of localized phototherapy and photodynamic therapy for psoriasis: a systematic review and meta-analysis. Photodermatol Photoimmunol Photomed. 2015;31(1):5-14. doi: 10.1111/phpp.12092

7. Hong J, Malick F, Sivanesan P, Koo J. Expanding the use of the 308 $\mathrm{nm}$ excimer laser for treatment of psoriasis. Pract Dermatol (Suppl). 2007; S13-6.

8. Tang YJ, Xu WW, Liu XM, Zhang RZ, Xu CX, Xu B, Cheng S, Liu Q. Self-control study of combination treatment of $308 \mathrm{~nm}$ excimer laser and calcipotriene ointment on stable psoriasis vulgaris Int J Clin Exp Med. 2014 15;7(9):2844-50.

9. Bianchi B, Campolmi P, Mavilia L, Danesi A, Rossi R, Cappugi P. Monochromatic excimer light (308 nm): an immunohistochemical study of cutaneous $\mathrm{T}$ cells and apoptosis-related molecules in psoriasis. J Eur Acad Dermatol Venereol. 2003;17(4):408-13.

10. Gattu S, Pang ML, Pugashetti R, Malick F, Hong J, Bowers E, et al. Pilot evaluation of supra-erythemogenic phototherapy with excimer laser in the treatment of patients with moderate to severe plaque psoriasis. J Dermatolog Treat. 2010;21(1):54-60. doi: 10.3109/09546630903203756..
11. Kardorff B. Honig d'Orville, I, Elections M, Kardorff M, Dorittke, P. Combination therapy of psoriasis vulgaris with the 308$\mathrm{nm}$ excimer laser and calcipotriol: Preliminary review. Current Dermatology. 2003;29(6):236-239.

12. Bonis B, Kemény L, Dobozy A, Bor Z, Szabó G, Ignacz F. 308 nm UVB excimer laser for psoriasis. The Lancet. 1997;350(9090):1522.

13. Menter A, Korman NJ, Elmets CA, Feldman SR, Gelfand JM, Gordon $\mathrm{KB}$. Guidelines of care for treatment of psoriasis with phototherapy and photochemotherapy. J Am Acad Dermatol. 2010;62(1):114-35.

14. Debbaneh MG, Levin E, Sanchez Rodriguez R, Leon A, Koo J, Rosenblum MD. Plaque-based sub-blistering dosimetry: Reaching PASI-75 after two treatments with 308-nm excimer laser in a generalized psoriasis patient. J Dermatolog Treat. 2015;26(1):45-8. doi: 10.3109/09546634.2013.879094.

15. Lavker RM, Gerberick G, Veres D, Irwin CJ, Kaidbey KH. Cumulative effects from repeated exposures to suberythemal doses of UVB and UVA in human skin. J Am Acad Dermatol. 1995;32(1):53-62 .

16. Asawanonda P, Anderson RR, Chang Y, Taylor CR. 308-nm excimer laser for the treatment of psoriasis: a dose-response study. Arch Dermatol. 2000;136(5):619-24.

17. Cochrane Training [Internet]. Cochrane Handbook for Systematic Reviews of Interventions | Cochrane Training. [cited 2016May11]. Available from: https://training.cochrane.org/grade-approach

18. Dong J, He Y, Zhang X, Wang Y, Tian Y, Wang J. Clinical efficacy of flumetasone/salicylic acid ointment combined with 308-nm excimer laser for treatment of psoriasis vulgaris. Photodermatol Photoimmunol Photomed.2012;28(3):133-6. doi: 10.1111/j.1600-0781.2012.00654.x.

19. Osmancevic A, Gillstedt M, Wennberg A, Larkö O. The Risk of Skin Cancer in Psoriasis Patients Treated with UVB Therapy. Acta Dermato Venereologica. 2014;94(4):425-30.

The Acta Medica Philippina is now accepting original scientific papers, review articles and case reports for its upcoming issues. Please follow the format for submission as indicated in the "Instructions to Authors" elsewhere in the journal. All papers received shall be properly acknowledged. For inquiries and submission of proposals, please e-mail us at actamedicaphilippina@yahoo.com 